The hospice employed an autogenic psychotherapist to treat a patient who was in a chronic state of stress. It was identified that stress management would have a profound effect on the patient's overall condition. Teaching sessions were provided to staff enabling them to use techniques that have shown positive symptom management results.

Conventional practice is to treat with medication - analgesia and anxiolytics. The aim was to introduce a variety of techniques giving both patients and their carers strategies to use to help relieve symptoms of anxiety and pain within palliative care.

Training sessions included experiential learning and reflective practice, taught staff to use simple breathing exercises, progressive muscular relaxation, guided imagery and visualisation techniques.

Staff discovered a powerful tool to help patients and their carers to take control of their own situation, using non pharmacological interventions to give control over physical responses to symptom management.

Using a toolkit of techniques, patients are supported to use the techniques on a face-to-face basis, using technology with CDs, and personalised recordings that they can use when and where required.

Enabling patients when they feel most out of control has shown improvements in psychological distress and physical symptom control. Staff have felt more empowered and able to introduce accessible psychological interventions alongside conventional pharmacological prescriptions.

\section{P-130 A TABOO SYMPTOM? ASSESSING MALODOUR FROM MALIGNANT FUNGATING WOUNDS}

${ }^{1}$ Mandy Stamper, ${ }^{2}$ Laura Green, ${ }^{2}$ Louise Wem. ' Overgate Hospice, Elland, Halifax, West Yorkshire; ${ }^{2}$ University of Bradford, Bradford, UK

\subsection{6/bmispcare-2017-hospice.155}

Background Symptom control of malodour in patients with Malignant Fungating Wounds (MFW) can be challenging as there is little evidence or guidelines. Malodour can be a taboo in society. Human response to smell is complex. A literature review showed that malodour is a major concern to patients, families and staff. As a nurse working in a hospice, I wanted to look at how we could develop a standard for assessing malodour.

Aim My presentation shares my experience in setting a standard for auditing the assessment of the symptom of malodour for patients with MFWs in a UK hospice. I chose this area because malodour from MFWs is a challenge in practice.

Methods I carried out a literature search to see what evidence and guidelines there were to inform my audit standard. I analysed the information and reflected on my 11 years' experience as a hospice nurse.

Results Patients with the symptom of malodour from MFW are affected physically, emotionally and socially. The symptom also affects their family and friends and can be distressing for other patients and staff. I felt the practice of assessing malodour at the hospice could improve so would start a clinical audit with my standard that;

'All patients that are admitted to the hospice with a malignant fungating wound will have the symptom of malodour holistically assessed to support the development of a patientcentred plan of care aimed at improving quality of life.'

Conclusions I was then able to audit the assessment of malodour as a basis for change in our practice aims at improving the management of this distressing symptom. The results have informed my leading a multidisciplinary group to devise a holistic assessment tool for assessing the symptoms of malignant fungating wounds.

\section{P-131 HOSPITAL COMPLIANCE WITH NICE GUIDANCE - INITIATING STRONG OPIOIDS FOR PAIN IN PALLIATIVE CARE}

Deborah Lam, Jennie Pickard, Mona Shalabi, Sophie Harrison, Samantha Kay. University Hospital of South Manchester NHS Foundation Trust, Manchester, UK

10.1136/bmjspcare-2017-hospice. 156

Background When strong opioids are initiated for pain in patients receiving palliative care, practice varies. As a result, there is a risk that patients will receive either sub-therapeutic or potentially toxic doses of opioids. Choice of formulation is important from a patient concordance and cost perspective. In May 2012, the National Institute for Health and Clinical Excellence (NICE) published guidelines on initiating strong opioids for adults with advanced and progressive disease ('Opioids in palliative care: safe and effective prescribing of strong opioids for pain in palliative care of adults'). We reviewed the prescribing practice on hospital wards and assessed our compliance with the guidance and considered the barriers to compliance.

Aim To assess compliance with NICE guidance when initiating strong opioids for pain in adults with a life-limiting illness.

Methods We identified 20 patients with a life limiting illness and that were initiated on strong opioids for pain during their current hospital admission. We reviewed the medical notes and prescriptions measuring prescribing practice against 10 standards outlined by NICE. The patients were from 12 wards including medical and surgical wards. The data was collected over three months.

Results Out of the 10 standards, compliance was 100\% in three standards, $50 \%$ or above in six standards, and less than $50 \%$ in one standard.

Conclusions The results highlighted areas for improvement. The main barrier appeared to be lack of awareness of the guidance. Due to the high turnover of medical staff, continued education is crucial. We have developed an e-learning module, highlighting the guidance, which will be part of the Trust's mandatory training. The Trust is implementing electronic prescribing and endeavours to include alerts when opioids are prescribed to encourage compliance. With improved compliance, prescribing practice will be safer and the management of pain in our patients will be more effective.

\section{P-132 APPROPRIATE AND INAPPROPRIATE USE OF NALOXONE IN PALLIATIVE CARE - TWO CASE REPORTS FROM A HOSPITAL}

Deborah Lam, Jennie Pickard, Sophie Harrison, Samantha Kay. University Hospital of South Manchester NHS Foundation Trust, Manchester

10.1136/bmjspcare-2017-hospice. 157 